\title{
Low-Temperature Magnetoresistance of a Disordered Metal
}

\author{
T. F. Rosenbaum, ${ }^{(a)}$ R. F. Milligan, ${ }^{(b)}$ G. A. Thomas, P. A. Lee, \\ T. V. Ramakrishnan, (c) and R. N. Bhatt \\ Bell Laboratories, Murray Hill, New Jersey 07974 \\ and \\ K. DeConde, H. Hess, and T. Perry \\ Joseph Henry Laboratory, Princeton University, Princeton, New Jersey 08544 \\ (Received 21 May 1981)
}

\begin{abstract}
A contribution to the magnetoresistance is observed at temperatures below $100 \mathrm{mK}$ in bulk metallic Si:P that is unanticipated within theoretical analyses of localization. This contribution is positive, approximately independent of sample orientation, and varying roughly as the square root of the applied field. An analysis of Coulomb interactions including spin splitting is presented which, when combined with localization, describes the magnetoresistance.
\end{abstract}

PACS numbers: 72.15.Gd, 71.45.-d, 71.50.+t

We have studied the magnetoresistance of bulk $\mathrm{Si}$ : $\mathrm{P}$ at temperatures below $100 \mathrm{mK}$ to investigate the relative importance of localization ${ }^{1}$ and Coulomb interaction $s^{2}$ in the transport properties of disordered metals. At higher temperatures, previous extensive studies ${ }^{3-7}$ show effects that have been interpreted as arising from either donor spins $^{3-7}$ or localization. ${ }^{8}$ At lower temperatures, we find that these negative contributions to the magnetoresistance are dominated by a positive one. We present an analysis of Coulomb interactions including electron-spin splitting which provides a description of the high-field behavior, its temperature dependence, and the observed crossover to the usual $H^{2}$ behavior at low field. We suggest that this Coulomb contribution be combined with that from localization to explain the change in sign of the magnetoresistance.
We have made four-probe resistance measurements of metallic, three-dimensional samples of uncompensated $\mathrm{Si}: \mathrm{P}$ as a function of temperature, $T$, magnetic field, $H$, and donor density, $n$, at a frequency of $\sim 10 \mathrm{~Hz}$. Temperatures down to 3 $\mathrm{mK}$ were reached by use of a dilution refrigerator with an adiabatic demagnetization stage.

As shown in Fig. 1, we find both positive and negative contributions to the magnetoresistance at $T \simeq 30 \mathrm{mK}$, with the positive component dominating as the concentration $n$ approaches the critical density $n_{c}=3.74 \times 10^{18} \mathrm{~cm}^{-3}$ and as the $(T=0$, $H=0$ ) conductivity $\sigma(0)$ drops with respect to Mott's characteristic value $\sigma_{\min }$. [For $\mathrm{Si}: \mathrm{P}$, $\sigma_{\text {min }}=20(\Omega \mathrm{cm})^{-1}$.] As discussed by Kawabata, ${ }^{8}$ a localization model accounts qualitatively for the negative magnetoresistance observed ${ }^{3-7}$ at higher temperatures $(T \sim 1 \mathrm{~K})$. He predicts a contribution $^{9}$ of the form

$$
\text { for } H \gg H_{c} \text {, }
$$

where $\Delta \rho / \rho(0,0)=[\rho(H, T)-\rho(0, T)] / \rho(0,0), A_{l}$ $=-0.918 \rho(0,0)$ for $H$ in kilo-oersteds and $\rho$ in ohm centimeters, and $4 e H_{c} / \hbar c \equiv\left(D \tau_{\text {in }}\right)^{-1}$. Here, $\rho(0,0) \equiv 1 / \sigma(0), D$ is the diffusion constant, and $\tau_{\text {in }}$ is the inelastic scattering time. We evaluate $D$ for Si:P using the formula $3.2 \hbar^{2} \sigma(0) /\left[e^{2} m^{*}\right.$ $\left.\times\left(\nu^{2} n\right)^{1 / 3}\right]$, where $m^{*}=0.26 m_{e}$ is the effective mass and $\nu=6$ is the number of valleys. For reasonable estimates ${ }^{8}$ of $\tau_{\text {in }}, H_{c}$ is small enough that Eq. (1) describes the negative contribution due to localization over the range of $H$ we have studied. The positive component of the magnetoresistance remains unexplained within this model. Ootuka et al. ${ }^{7}$ have qualitatively discussed scattering from random impurity spins which can produce both positive and negative contributions, although they appear to be small. Altshuler and Aronov ${ }^{10}$ have calculated some contributions within the Coulomb interactions picture, but a systematic analysis ${ }^{11}$ shows that these are canceled by other terms.

We have considered Coulomb interactions with spin splitting to explain the positive magnetoresistance. As discussed previously, ${ }^{2,12}$ the Coulomb-interactions picture yields a low-temperature resistivity given by

$$
\rho=\rho(0,0)-\alpha\left(\frac{4}{3}-2 F\right) \sqrt{T},
$$

where $\alpha=0.025\left[T_{\mathrm{F}}\left(m^{*} D / \hbar\right)^{3}\right]^{-1 / 2} \rho(0,0)$ and $T_{\mathrm{F}}$ is 
the Fermi temperature. The $\frac{4}{3}$ term in Eq. (2) comes from the exchange interaction among the electrons in the Fermi sea while the $2 F$ term comes from the Hartree interaction. (The factor of 2 is from the spin directions and $F$ is a func$\operatorname{tion}^{11,12}$ of $\kappa / 2 k_{\mathrm{F}}$, where $\kappa$ and $k_{\mathrm{F}}$ are the screening and Fermi wave vectors, respectively.) In the presence of $H$ the lowest unoccupied spin-up electron is separated from the highest occupied

$$
\rho(H, T)=\rho(0,0)-\alpha\left(\frac{4}{3}-F\right) T^{1 / 2}+\alpha F T^{1 / 2} G(h) / G(0),
$$

where

$$
G(h)=\int_{0}^{\infty} d \omega \frac{\partial^{2}}{\partial \omega^{2}}\left(\frac{\omega}{e^{\omega}-1}\right)\left[(\omega+h)^{1 / 2}+(|\omega-h|)^{1 / 2}\right]
$$

and $h=g \mu_{\mathrm{B}} H / k T$. We find that $G(h) / G(0) \approx 0.77 \sqrt{h}$ for $h \gg 1$ and $G(h) / G(0) \approx 1+O\left(h^{2}\right)$ for $h \ll 1$. From Eq. (3) we see that the magnetoresistance is always positive and that the last term approaches $0.77 \alpha \boldsymbol{F}\left(g \mu_{\mathrm{B}} H / k\right)^{1 / 2} \equiv A_{c} \rho(0,0) H^{1 / 2}$ independent of $T$ for $g \mu_{\mathrm{B}} H \gg k T$. In that same limit the only tem-

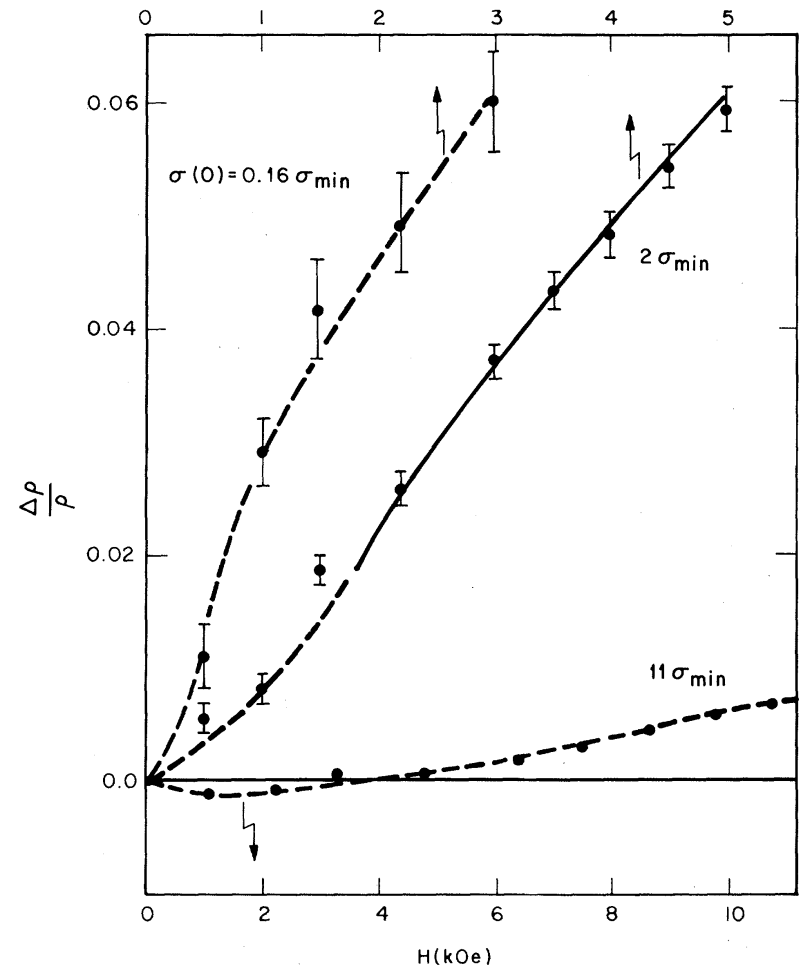

FIG. 1. $\Delta \rho / \rho$ vs $H$ for three samples characterized by $\left\{\sigma(0)=0.16 \sigma_{\min }, m_{e} D / \hbar=0.04, T=34 \mathrm{mK}\right\}$, and $\left\{2 \sigma_{\min }, 0.4,32 \mathrm{mK}\right\}$, both upper scale, and $\left\{11 \sigma_{\min }, 2\right.$, $32 \mathrm{mK}\}$, lower scale. Dashed line for $0.16 \sigma_{\min }$ is a fit by the empirical equation $\Delta \rho / \rho=\left(1 / C H^{2}+1 / E H^{1 / 2}\right)^{-1}$; for $2 \sigma_{\mathrm{min}}$ and $11 \sigma_{\mathrm{min}}$ it is a guide to the eye. Solid line is calculated with use of Eq. (5). Error bars for $11 \sigma_{\mathrm{min}}$ are the size of the data points and are shown more clearly in Fig. 3. spin-down electron by an energy $g \mu_{\mathrm{B}} H$. We recall that the $\sqrt{T}$ dependence in $\mathrm{Eq}$. (2) comes from the singular correlation between wave functions nearby in energy. For the Hartree interaction between opposite spins this singularity is now cut off at $g \mu_{\mathrm{B}} H$ instead of $k T$. On the other hand, the exchange term and the Hartree term between parallel spins are unaffected. The resistivity in a field thus becomes ${ }^{11}$

perature dependence comes from the second term in Eq. (3) and the coefficient of $\sqrt{T}$ is always negative, for the single-valley, free-electron case ${ }^{12}$ where $0<F<1$. For $H=0 \mathrm{Eq}$. (3) reduces to Eq. (2). In terms of $\sigma(T) \equiv 1 / \rho(0, T)$ we have $\sigma(T)$ $=\sigma(0)+m \sqrt{T}$, where $m \equiv \alpha\left(\frac{4}{3}-2 F\right) / \rho^{2}(0,0)$, as previously discussed. ${ }^{12}$

We suggest that a reasonable approximation is to combine the localization and Coulomb-interaction models, so that the low-temperature magnetoresistance for $h \gg 1$ takes the form

$$
\Delta \rho / \rho(0,0)=A H^{1 / 2}-B T^{1 / 2} \text {, }
$$

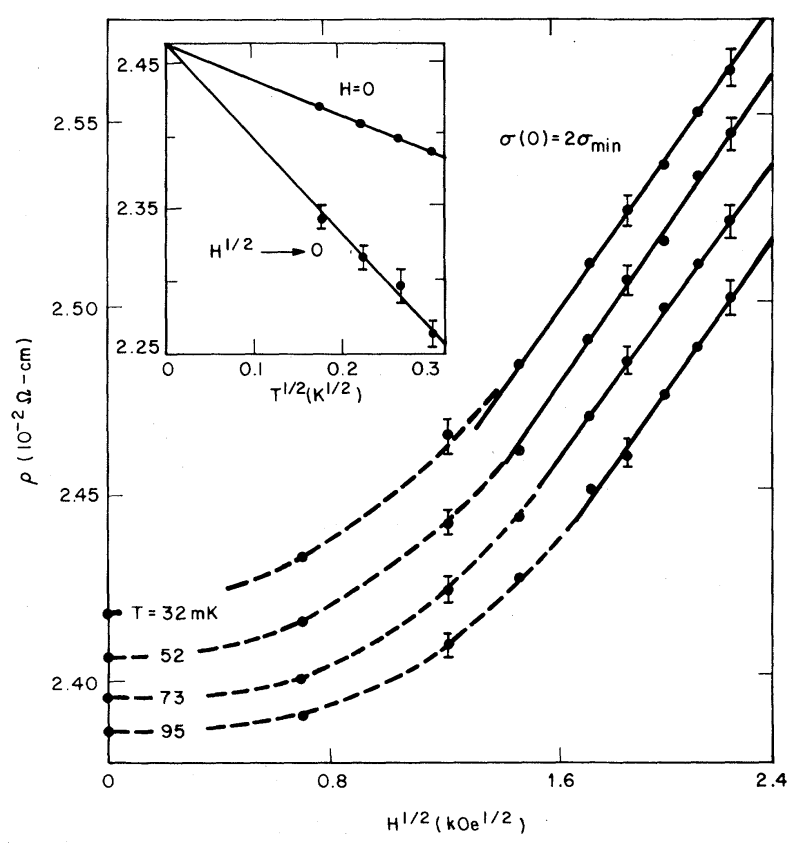

FIG. 2. $\rho$ vs $H^{1 / 2}$ at four temperatures for a sample of Si:P with $\sigma(0)=2 \sigma_{\min }$. Solid lines show $\rho$ to be linear in $H^{1 / 2}$ at high fields. Extrapolated values of $\rho$ for $H^{1 / 2} \rightarrow 0$, as well as the values for $H=0$, are plotted against $T^{1 / 2}$ in the inset. 
where theory predicts $A=A_{c}+A_{l}$ and $B=\alpha F /$ $\rho(0,0)$. A combination of positive and negative contributions is necessary to explain the sign change observed for the sample with $\sigma(0)=11 \sigma_{\text {min }}$ in Fig. 1. Since $A_{c}$ is proportional to $\rho(0,0)^{3 / 2}$ we expect it to dominate near the transition, and Fig. 1 also shows this positive increase near $n_{c}$. All three samples show high-field magnetoresistance proportional to $H^{1 / 2}$. For the $\sigma(0)=11 \sigma_{\text {min }}$ sample, $A_{c}$ and $A_{l}$ nearly cancel and fields up to $\sim 10 \mathrm{kOe}$ were required to show the $H^{1 / 2}$ effect.

Figure 2 shows $\rho(H, T)$ plotted against $H^{1 / 2}$ for the $2 \sigma_{\text {min }}$ sample at four temperatures from 32 to $95 \mathrm{mK}$, with the results for $H$ parallel and perpendicular to the electric field equal ${ }^{9}$ within experimental error. The solid lines show the linear behavior down to $H$ of the order of the expected crossover region: $h \sim 1$. Equations (2) and (3) predict that both $\rho(0, T)$ and the extrapolated values of the solid lines to $H^{1 / 2}=0$ should be linear in $T^{1 / 2}$. This behavior is shown in the inset of Fig. 2, where both curves are constrained to go through the same value of $\rho(0,0)$. From the average of the slopes of the solid lines in Fig. 2 we can obtain an experimental value for $A$ in Eq. (5). We get $A=0.044 \pm 0.004 \mathrm{kOe}^{-1 / 2}$. From the fit in the inset we get $B=0.26 \pm 0.06 \mathrm{~K}^{-1 / 2}$. The theoretical evaluations of $A_{c}$ and $A_{l}$ are uncertain for this sample both because of the unknown scattering rates ${ }^{12}$ that enter $F$ and because the sample has a dimensionless diffusion constant $m_{e} D / \hbar$ $=0.4$, rather than $m_{e} D / \hbar \gg 1$ as assumed theoretically. However, we can make a less ambiguous comparison of theory and experiment by assuming Eq. (1) for $A_{l}$ and considering the ratio $B / A_{c}$, in which $\alpha F$ cancels. Theoretically $B / A_{c}$ $=1.3\left(\mathrm{k} / \mathrm{g} \mu_{\mathrm{B}}\right)^{1 / 2}=3.5(\mathrm{kOe} / \mathrm{K})^{1 / 2}$ for $g=2$, independent of other sample parameters. Combining $A_{\exp }$ and Eq. (1) for $A_{l}$, we find $B / A_{c}=0.26 /(0.044$ $+0.023)=4 \pm 1(\mathrm{kOe} / \mathrm{K})^{1 / 2}$, in agreement with theory.

As shown in Fig. 1, the positive magnetoresistance is large for the $0.16 \sigma_{\mathrm{min}}$ sample. Qualitatively, a large effect is predicted, but we make no quantitative comparison because $m_{e} D / \hbar=0.04$. The dashed line through the data is a fit to the empirical form $\Delta \rho / \rho(0,0)=\left(1 / C H^{2}+1 / E H^{1 / 2}\right)^{-1}$, where $C$ and $E$ are experimentally derived constants. We find that the $H^{2}$ term has grown by $\sim 10^{2}$ over that in the $2 \sigma_{\text {min }}$ sample; nevertheless, there is a qualitative similarity between these two samples even though one has $\sigma(0)=0.16 \sigma_{\min }$.

The most metallic sample shown in Fig. 1 provides a better test of the theoretical parameters

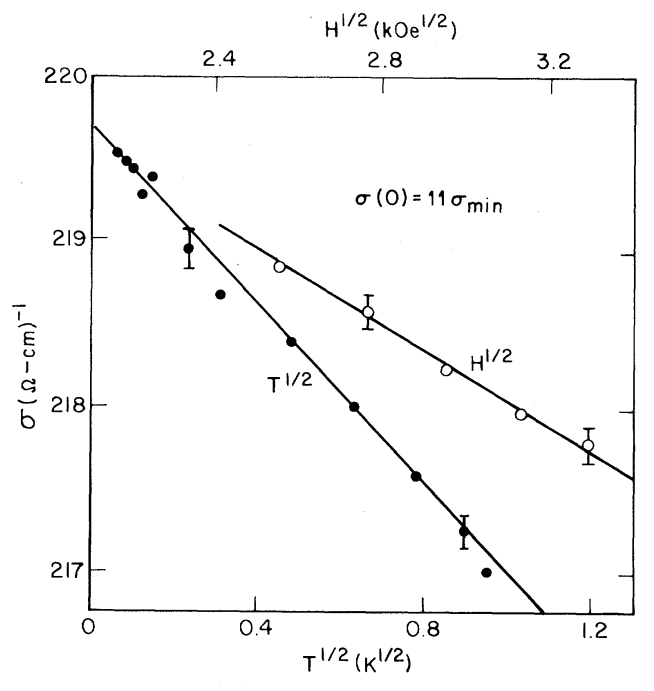

FIG. 3. $\sigma$ vs $T^{1 / 2}(H=0)$, solid circles, and $\sigma$ vs $H^{1 / 2}(T=32 \mathrm{mK})$, open circles, for $11 \sigma_{\mathrm{min}}$ sample. Slopes allow determination of $\alpha$ and $F$ separately with us of Eqs. (1) - (4)。

in Eqs. (1)-(4) since it has $m_{e} D / \hbar \sim 2$. Figure 3 shows the conductivity at $H=0$ plotted versus $T^{1 / 2}$ for $T=3 \mathrm{mK}$ to $1 \mathrm{~K}$, as well as $\sigma$ plotted versus $H^{1 / 2}$ at $T=32 \mathrm{mK}$ for $g \mu_{\mathrm{B}} H \gg k T$. The solid lines through the data are fits which determine the experimental values $A=(6.6 \pm 1.2) \times 10^{-3} \mathrm{kOe}^{-1 / 2}$ and $m=-2.8 \pm 0.3\left(\Omega \mathrm{cm} \mathrm{K}^{1 / 2}\right)^{-1}$. Theoretically, Eq. (1) gives $A_{l}=-4.2 \times 10^{-3}$ and Eqs. (2)-(4) give $m$ $=-0.21$ and $A_{c}=0.65 \times 10^{-3} \mathrm{kOe}^{-1 / 2}$, if we use the single-valley free-electron formula ${ }^{12}$ for $F$. If we assume Eq. (1) to be exact, we can combine our experimental values of $A$ and $m$ to evaluate $F$ and $\alpha$ separately: $F=0.80$ and $\alpha=2.2 \times 10^{-4} \Omega$ $\mathrm{cm} / \mathrm{K}^{1 / 2}$. With this assumption the theoretical values $F=0.84$ and $\alpha=0.25 \times 10^{-4} \Omega \mathrm{cm} / \mathrm{K}^{1 / 2}$ indicate numerical disagreement in $\alpha$ and that some further theoretical analysis is needed.

In conclusion, we have studied the magnetoresistance of three metallic $\mathrm{Si}: \mathrm{P}$ samples with a range of $(T=0, H=0)$ conductivities from $\frac{1}{6} \sigma_{\mathrm{min}}$ to $11 \sigma_{\text {min }}$. A positive contribution, based on Coulomb interactions with spin splitting, added to the negative localization contribution, qualitatively explains the results. The relative strengths of the two contributions change rapidly with donor concentration near the transition.

We would like to thank J. B. Mock and P. H. Schmidt for technical assistance and A. Kawabata for helpful discussions. This work was partially supported by Grant No. DMR-79-04141 from the National Science Foundation. 


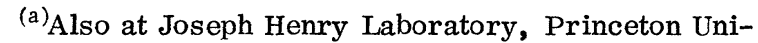
versity, Princeton, N. J. 08544.

(b) Also at Muhlenberg College, Allentown, Pa. 18104.

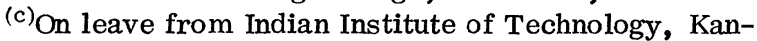
pur, India.

${ }^{1}$ E. Abrahams, P. W. Anderson, D. C. Licciardello, and T. V. Ramakrishnan, Phys. Rev. Lett. $\underline{42}, 673$ (1979), and references therein.

${ }^{2}$ B. L. Altshuler, A. G. Aronov, and P. A. Lee, Phys. Rev. Lett. 44, 1288 (1980); B. L. Altshuler, D. Khmelnitzkii, A. I. Larkin, and P. A. Lee, Phys. Rev. B 22, 5142 (1980).

${ }^{3}$ See M. N. Alexander and D. F. Holcomb, Rev. Mod. Phys. 40, 815 (1968).

${ }^{4}$ N. F. Mott, The Metal-Insulator Transition (Taylor and Francis, London, 1974).

${ }^{5}$ W. Sasaki, J. Phys. Soc. Jpn. Suppl. 49, 31 (1980).

${ }^{6}$ W. Sasaki, Philos. Mag. B $42,725(1981)$.

${ }^{7}$ Y. Ootuka, S. Kobayashi, S. Ikehata, W. Sasaki, and J. Kondo, Solid State Commun. 30, 169 (1979).

${ }^{8}$ A. Kawabata, Solid State Commun. 34, 431 (1980), and J. Phys. Soc. Jpn. 49 , Suppl. A, 375 (1980).

${ }^{9}$ Equation (1) corrects the factor equal to the number of valleys in Kawabata's calculation (Ref. 8), which has been shown by H. Fukuyama, J. Phys. Soc. Jpn. $\underline{49}$,
$649(1980)$, to be absent if intervalley scattering rates exceed the inelastic collision rates. Kawabata also calculates that the prefactor can increase by from $15 \%$ to $35 \%$ in Si because of mass anisotropy, dependent upon the orientation of the crystallographic axis relative to $\overrightarrow{\mathrm{H}}$ and $\overrightarrow{\mathrm{E}}$. Changes in the prefactor of a similar magnitude could be expected as well due to anisotropy in relaxation rates (see, e.g., R. N. Bhatt and T. V. Ramakrishnan, to be published, for the corresponding twodimensional calculation).

${ }^{10}$ B. L. Altshuler and A. G. Aronov, Solid State Commun. 38,11 (1981).

${ }^{11} \mathrm{P}$. A. Lee and T. V. Ramakrishnan, to be published.

${ }^{12}$ T. F. Rosenbaum, K. Andres, G. A. Thomas, and P. A. Lee, Phys. Rev. Lett. 46, 568 (1981). The theoretical coefficient, $\alpha$, in this paper is too large by a factor of 4. This error has been corrected here in Eq. (2) and in Ref. 11. The function $F$, which is $(1 / x)$ $x \ln (1+x)$ for free electrons in the Thomas-Fermi approximation with a single valley, where $x=\left(2 k_{\mathrm{F}} / K\right)^{2}$, should be modified for Si:P. Depending on the ratio of $k T$ and the intervalley scattering rate, $F$ may be increased or decreased by a factor (up to 6) that is unknown because the intervalley rate is not accurately known.

\title{
First-Order Magnetic Phase Transitions in Fe
}

\author{
Kristl B. Hathaway \\ Naval Surface Weapons Center, White Oak, Silver Spring, Maryland 20910 \\ and
}

Gary A. Prinz

Naval Research Laboratory, Washington, D. C. 20375

(Received 17 August 1981)

\begin{abstract}
The first observation of a first-order phase transition in the magnetization of $\mathrm{Fe}$ in an applied field is reported. The samples were epitaxially grown (110) Fe films on GaAs. Mean-field theory, with the inclusion of uniaxial anisotropy and the constraint that $\overrightarrow{\mathrm{M}}$ lie in the plane of the film, gives excellent agreement with the observed transition. The relationship of this transition to the predictions of the three-state Potts model for cubic ferromagnets is discussed.
\end{abstract}

PACS numbers: $75.30 . \mathrm{Kz}, 75.50 . \mathrm{Bb}, 75.10 .-\mathrm{i}$

Cubic ferromagnetic iron is predicted by meanfield theory to exhibit a first-order transition in magnetization as a function of applied field along a [111] crystallographic direction in a single crystal. ${ }^{1,2}$ There is no evidence of this transition in the original magnetization measurements of Honda and $\mathrm{Kaya}^{3}$ or of Williams ${ }^{4}$ on single crystals of $3.8 \% \mathrm{Si}$ in Fe. There is observed instead a rapid increase of slope as $H \rightarrow H_{A}$, the anisot- ropy field, with a discontinuity in the first derivative at $H=H_{A}$. More recently an attempt was made to observe the predicted transition in singlecrystal $[111]$ iron whiskers. ${ }^{5}$ That study also failed to reveal any discontinuity, yielding results very similar to the earlier work.

Renewed interest in observing this transition has been stimulated by the identification of cubic ferromagnets as realizations of the three-state 